\title{
STUDIES IN FLICKERING
}

\author{
W. F. WELSH ${ }^{1}$, JANET H. WOOD ${ }^{1}$, K. HORNE ${ }^{2}$ \\ 1 Keele University ${ }^{2}$ University of St.Andrews
}

\begin{abstract}
Flickering is a poorly understood phenomenon associated with accretion processes, but this does not suffice to make it interesting. Why then should we bother studying this 'noise'? Three reasons come to mind: (i) flickering is a fundamental signature of accretion, to the point of being a necessary characteristic (if it doesn't flicker, it's not a CV); (ii) energetically, flickering is not a small effect and can contribute up to a few tens of percent of the total luminosity of the system (hence it is often more luminous than the entire secondary star); (iii) flickering is inherently a time-dependent phenomenon and it is hoped that it can provide clues to the nature of the disc viscosity, something that time-independent theory cannot provide.
\end{abstract}

\section{Study 1: Eclipse mapping the flickering}

To determine where the flickering originates, it is best to examine an eclipsing system. Because the amplitude of the flickering decreases during eclipse, it has been known for a long time that the flickering is associated with the white dwarf, disc and/or hot spot. By use of the eclipse mapping method [3] the mean eclipse light curve can be used to spatially resolve structure in the disc. The concept is trivially extended to mapping the flickering. The difficulty lies in determining the eclipse light curve of the flickering.

Two possible methods are available for determining the flickering light curves, which we will call the 'single' and the 'ensemble' methods. In both cases, many light curves are needed to remove sensitivity to individual flickers and to get a statistically significant result. In the absence of any changes in the state of the CV (i.e. the steady plus random variations are statistically stationary), these methods are identical. Obviously things like dwarf nova outbursts violate this criterion, but changes in observing conditions (e.g. different detector sensitivity or bandpasses) can also cause problems. To minimize these contaminating effects, it is best to observe all the light curves as close together in time as possible. The 'single' method (championed by Bruch [2]) computes the deviation of the observed light curve from a running mean. This is done for many light curves and the results

A. Evans and J. H. Wood (eds.), Cataclysmic Variables and Related Objects, 29-32.

(C) 1996 Kluwer Academic Publishers. Printed in the Netherlands. 
averaged. Its main disadvantage is the difficulty of separating the rapid flickering fluctuations from the rapid variations due to the eclipse itself. Just as flickering contaminates the mean eclipse light curve when studying the steady disc, so too will the disc eclipse contaminate the study of the flickering; in the end, one must exercise extreme care. Horne \& Stiening [4] laid the foundation of the 'ensemble' method: the flickering light curve is defined as the rms of the residuals of the individual light curves relative to the mean light curve at each orbital phase. The advantages of this method are (i) because many observations are used the mean is well defined and so there is no need to approximate it (via a running mean or spline or polynomial fit); (ii) each orbital phase is treated independently (thus there are no correlations between adjacent bins). The major disadvantage is the sensitivity to violations of stationarity. This latter problem is very serious, as it requires that there are no substantial changes in the disc structure (or detector/observing conditions) over the course of obtaining the data. If this is violated, the flickering eclipse light curves can be misleading (one is seeing the effects of long-term structural changes in the disc, or instrumental effects, and not flickering). Luckily it is easy to test if stationarity has been violated and so one knows when compensations for non-stationarity are needed. Unfortunately, these corrections are not easy to determine and the final results are sensitive to any assumptions that go into them. However, with care they can be derived (e.g. [1]) and the ensemble of light curves used. The alternative is to use only those light curves which lie within some tolerance of the mean. We prefer the ensemble method because it has no free parameters (no need to fit to the light curves). However the single method has the advantage of being able to cope more readily with observational non-stationarity. Both these methods will play important roles in determining the light curve of the flickering.

Analysis of the flickering in HT Cas, including eclipse mapping, is in progress [5]. Preliminary results reveal that in the $U$-band, the flickering is highly concentrated toward the center of the disc, but not confined to the immediate area around the white dwarf. The flickering amplitude increases towards the center faster than the mean (steady) light. The hot spot contributes a negligible amount of flickering.

\section{Study 2: The spectrum of the flickering Predictions of a simple model}

Assuming that flickering arises from dynamical time-scale processes, we have attempted to model the flickering by extending the standard steadystate accretion disc model in the simplest possible way, giving it a time dependency. This was accomplished by allowing small sinusoidal temperature variations about the local steady-state temperature:

$T(r, t)=T_{0}(r)\left[1+A \sin \left(2 \pi t / \tau_{\mathrm{Kep}}\right)\right]$, 
where $T_{0}(r) \propto r^{-3 / 4}$ and $\tau_{\text {Kep }}$ is the local Keplerian time-scale $\left(\propto r^{3 / 2}\right)$. Because each annulus in the disc radiates like a blackbody, and because a blackbody has an intensity maximum which varies inversely with temperature and has an exponential cutoff at wavelengths shorter than this (Wien's law), we have the following predictions about the power-spectrum: a highfrequency wavelength-independent cutoff, and a low-frequency wavelengthdependent roll over (roll over frequency $\propto \lambda^{-2}$ ). The main virtue of this model is its simplicity - the predictions are clear and unambiguous. By examining the power-spectrum at each wavelength ( $a$ 'power-spectrum spectrum'), we can test these predictions.
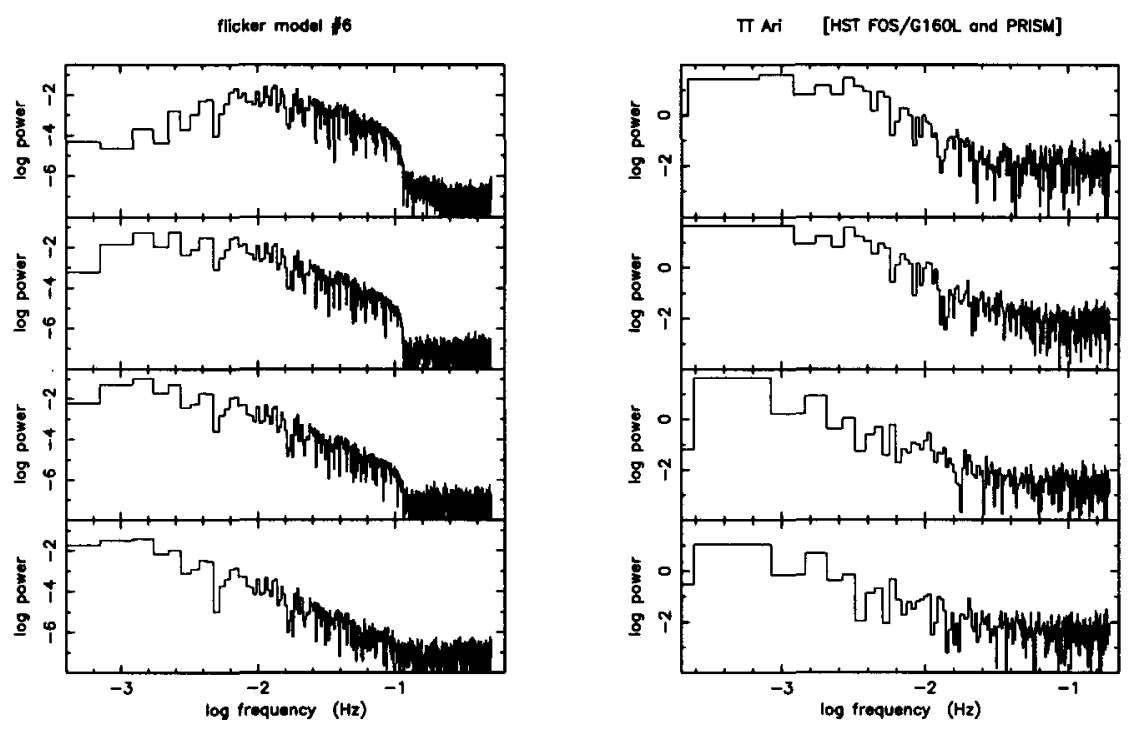

Figure 1. Left: Power spectra from flickering model at $\lambda 1000 \AA$ (top), 2000, 3000 and $6000 \AA$ (bottom). The high-frequency break occurs at the Keplerian time-scale of the innermost orbit $(\sim 10 \mathrm{~s})$ and is independent of wavelength. The wavelength-dependent low-frequency rollover occurs at time-scales longer than $\sim 4 \mathrm{~m}$. Right: TT Ari power spectra at $\lambda 1250-1400 \AA$ (top), 2000,3000 and $\sim 6000 \AA$ (bottom). No low-frequency rollover or high-frequency break is seen.

\section{A first look at some HST data on flickering}

High-speed HST FOS spectrophotometry of TT Ari and V603 Aql were examined and compared with these predictions. No low-frequency rollover is seen, and if any high-frequency cutoff exists, we have yet to dig it out of the noise. These preliminary results indicate that our simple model fails, or at least confines the parameters to an uncomfortably small region of parameter space. As the predictions of the model are inescapable, what 

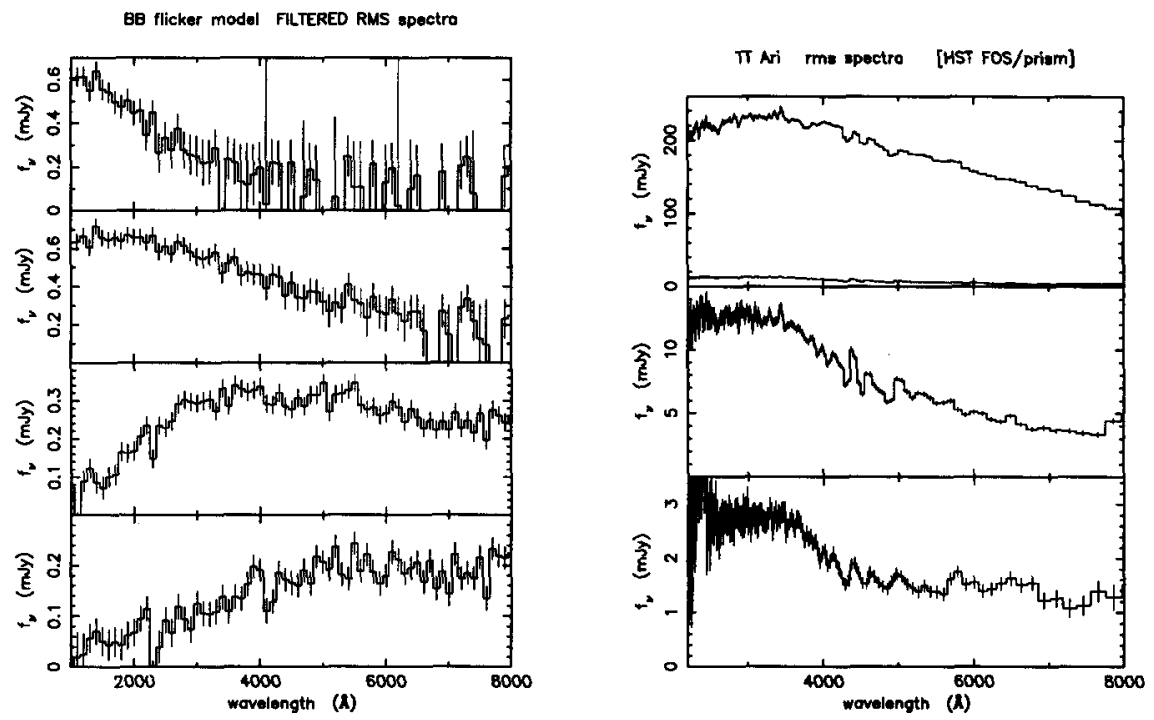

Figure 2. Left: Model flickering spectra (top to bottom): fastest flickering ( $\tau<100 \mathrm{~s}$ ), faster than $300 \mathrm{~s}$, slower than $300 \mathrm{~s}$, and the slowest flickering attainable from the $1000 \mathrm{~s}$ of simulated data. Note the strong time-scale dependence. Right (top to bottom): TT Ari mean, rms, and high-pass filtered rms spectra. The bottom panel shows the spectrum of the fastest flickering, revealing a large Balmer jump in emission.

has gone wrong? In essence, this failure indicates that our intuition about flickering is incorrect.

Delving deeper into the data, we find where our intuition breaks down by examining the actual spectrum of the flickering (determined by computing a 'debiased rms spectrum'). It appears that the spectrum of the fastest flickering contains a large Balmer jump in emission, indicating optically thin gas. Thus we have learned that the flickering cannot be treated as an extension of standard (optically thick) accretion disc theory.

\section{References}

1. Bennie, P.J., Hilditch, R.W., Horne, K., 1996, these proceedings, p33

2. Bruch, A., 1996, these proceedings, p35

3. Horne, K., 1985, MNRAS, 213, 129

4. Horne, K., Stiening, R.F., 1985, MNRAS, 216, 933

5. Welsh, W.F., Wood, J.H., 1995, in "Flares \& Flashes" - Proc. of IAU Colloquium 151, eds. J. Greiner, H.W. Duerbeck, R.E. Gershberg, Springer-Verlag, p300 